\title{
All-optical switching in lithium niobate directional couplers with cascaded nonlinearity
}

\author{
R. Schiek, Y. Baek, G. Krijnen, and G. I. Stegeman \\ Center for Research and Education in Optics and Lasers, University of Central Florida, 4000 Central Florida Boulevard, \\ Orlando, Florida 32816-2700
}

I. Baumann and W. Sohler

Angewandte Physik, Universität Paderborn, Warburger Strasse 100, D-33098 Paderborn, Germany

Received December 6, 1995

\begin{abstract}
We report on intensity-dependent switching in lithium niobate directional couplers. Large nonlinear phase shifts that are due to cascading detune the coupling between the coupler branches, which makes all-optical switching possible. Depending on the input intensity, the output could be switched between the cross and the bar coupler branches with a switching ratio of 1:5 and a throughput of $80 \%$. ( 1996 Optical Society of America
\end{abstract}

In a non-phase-matched second-order nonlinear interaction between a fundamental light wave and its second harmonic, the difference in the phase velocities leads to a periodic energy exchange with propagation distance between the interacting waves. Because the interaction is coherent, the phases are also affected, and a nonlinear phase shift accumulates with a propagation distance whose magnitude depends on the input fundamental intensity (cascaded nonlinearity). ${ }^{1}$ This implies that typical third-order phenomena, such as all-optical switching and soliton propagation, that are based on a nonlinear phase shift can also be implemented on the basis of cascading. ${ }^{2}$ This idea is particularly intriguing because there are many semiconductor and organic materials with second-order nonlinearities $>100 \mathrm{pm} / \mathrm{V}$. ${ }^{3}$ Therefore, under conditions of near phase matching, the nonlinear phase shift that is due to cascading can be orders of magnitude larger than phase shifts produced by the thirdorder nonlinearity (nonlinear refractive index) for the same input power. This should dramatically reduce the power levels required for a given application. Although both all-optical switching and solitonlike propagation based on the nonlinear refractive index have been successfully demonstrated during the past decade ${ }^{4,5}$ the first experiments to confirm the predictions that typical all-optical $\chi^{(3)}$ phenomena can be mimicked with the cascaded second-order nonlinearity were performed only a few years ago. Specifically, in $\mathrm{LiNbO}_{3}$ channel and slab waveguides the nonlinear phase shifts have been measured, ${ }^{6}$ one-dimensional spatial solitons have been observed in planar waveguides, ${ }^{7}$ and the nonlinear phase shift was used for what was to our knowledge the first demonstration of all-optical switching in a hybrid Mach-Zehnder interferometer. ${ }^{8}$

However, large cascading nonlinear phase shifts in the fundamental beam would be of limited value if they were inseparably accompanied by strong depletion of the fundamental. And, in general, this would seem to be the case, because the largest phase shifts occur near phase matching, where conversion of the fundamental into its second harmonic is strongest.
However, our previous experiments and calculations showed that varying the wave-vector mismatch in a specific way along the propagation direction, for example, by changes in the waveguide dimensions, the quasi-phase-matching period, or the temperature distribution (our case), leads to large nonlinear phase shifts commensurate with weak $(<10 \%)$ net conversion to second-harmonic generation (SHG) ${ }^{6-8}$ This has led us to test the feasibility of designing and fabricating waveguide-directional couplers in $\mathrm{LiNbO}_{3}$ for a first demonstration of all-optical switching owing to cascading in a fully integrated optics device. The results are reported here.

The experiments were performed in 50-mm-long titanium-indiffused symmetrical channel waveguide couplers with propagation along the $\mathrm{X}$ axis on a Y-cut $\mathrm{LiNbO}_{3}$ crystal. A series of waveguide couplers was produced by indiffusion of 43-nm-thick parallel titanium stripes with either a $13-$ or a $15-\mu \mathrm{m}$ width separated by $20-38 \mu \mathrm{m}$, at a temperature of $1060^{\circ} \mathrm{C}$ for $9 \mathrm{~h}$. The end faces were polished for end-fire coupling. The measurements were done with a Nd:YAG $Q$-switched, mode-locked pulsed laser and an electrooptic single-pulse extractor operated at a wavelength of $\lambda=1.32 \mu \mathrm{m}$ with a train of 90-ps FWHM pulses at a repetition rate of $800 \mathrm{~Hz}$. To characterize the nonlinear switching, we coupled radiation at $\lambda=1.32 \mu \mathrm{m}$ into only one coupler arm. The input power was measured. The output at both the fundamental and the harmonic wavelengths from both coupler branches was separated and measured independently. We used fast germanium detectors and boxcar integrators to measure the energies of the input and the output signals. To generate nonlinear phase shifts with cascading, Type I SHG was implemented from a fundamental $\operatorname{TM}_{00}(\omega)$ mode to the second harmonic $\mathrm{TE}_{00}(2 \omega)$ mode, which is phase matched near $345^{\circ} \mathrm{C}$ for our material, geometry, and wavelength. For this purpose the crystal was placed in an oven with a temperature-controlled stability of $\pm 25 \mathrm{mK}$ and a temperature profile appropriate for large phase shifts and small net SHG. ${ }^{6}$

We simulated the wave propagation in two linear coupled straight waveguides with cascading, 
using coupled-mode theory and taking into account the details of the experimental conditions. The modeling is based on graded-index profiles that are dependent on wavelength, polarization, and temperature and are determined by the indiffused titanium concentration profile. With a finite-difference program the modal fields and the temperature- and wavelength-dependent propagation constants are calculated. These data provide the parameters for the mode-coupling equations for the coupler with SHG present ${ }^{9}$ :

$$
\begin{aligned}
\frac{\mathrm{d} A_{1}}{\mathrm{~d} z}+j \beta_{F}(z) A_{1} & =-j \omega \epsilon_{0}\left[\left(J_{6} A_{1}+J_{7} A_{2}\right)\right. \\
& \left.+2 \chi^{(2)} J_{s 1} A_{3} A_{1}{ }^{*}+\chi^{(3)} J_{1} A_{1} A_{1} A_{1}{ }^{*}\right], \\
\frac{\mathrm{d} A_{3}}{\mathrm{~d} z}+j \beta_{\mathrm{SH}}(z) A_{3} & =-j 2 \omega \epsilon_{0}\left[\chi^{(2)} J_{s 1} A_{1} A_{1}\right] .
\end{aligned}
$$

Equations (1) describe the evolution of the fundamental mode power $\left|A_{1}\right|^{2} p_{0}$ and the secondharmonic mode power $\left|A_{3}\right|^{2} p_{0}$ in the bar (input) coupler branch in units of $p_{0}=1 \mathrm{~W}$ along the waveguide axis $(z)$ for $\mathrm{cw}$ excitation. Corresponding equations for $A_{2}$ and $A_{4}$ describe the fundamental and the second harmonic in the cross branch. $\beta_{F}(z)$ and $\beta_{\mathrm{SH}}(z)$ are the propagation constants of the fundamental and the second harmonic as a function of distance (reflecting the temperature dependence along the sample). The linear self- and cross-coupling integrals $J_{6}$ and $J_{7}$ for the fundamental modes and the nonlinear coupling integrals $J_{1}$ and $J_{s 1}$ are defined in Ref. 9. The nonlinear cross coupling can be neglected as well as both the third-order nonlinearity and the linear coupling for the second-harmonic modes. $\chi^{(2)}=\chi_{\mathrm{ZYY}}^{(2)}(2 \omega ; \omega, \omega)=-5.6 \mathrm{pm} / \mathrm{V}$ is the applicable second-order nonlinear susceptibility tensor element in $\mathrm{LiNbO}_{3}$. Losses of $0.17 \mathrm{~dB} / \mathrm{cm}$ for $\mathrm{TM}_{00}(\omega)$ and $0.35 \mathrm{~dB} / \mathrm{cm}$ for $\mathrm{TE}_{00}(2 \omega)$ are included in the simulations. We modeled the pulsed-light experiments with Eqs. (1) by averaging over the temporal pulse profile, assuming that the pulses were long enough that temporal dispersion could be neglected. For our experiments this approximation is valid.

On the basis of the simulations we found an optimized design and fabricated a series of directional couplers for operation with $\mathrm{TM}_{00}$ modes at $\lambda=1.32 \mu \mathrm{m}$. Half-beat-length couplers with a coupling length of $50 \mathrm{~mm}$ had waveguide separations of 32.0 and $32.5 \mu \mathrm{m}$ for branch widths of 15 and $13 \mu \mathrm{m}$, respectively. It was confirmed that the measured half-beat length did not change significantly over the 15-K temperature range of the experiments. From measurements of the SHG efficiency and tuning curves on single-channel waveguides fabricated in close proximity to the couplers, the calculated nonlinear coupling integral $J_{s 1}$ was confirmed and the effective temperature profile was deduced. The effective temperature profile can be approximated by

$$
\begin{aligned}
T= & T_{\mathrm{SET}}-\Delta T_{P} \\
& -0.35 \mathrm{~K}\left\{1-\exp \left[-\left(\frac{z / \mathrm{mm}-25}{2.733}\right)^{2}\right]\right\},
\end{aligned}
$$

with

$$
\begin{aligned}
& \Delta T_{p} / K \\
& = \begin{cases}\left(\frac{12.5-z / \mathrm{mm}}{5}\right)^{2} & \text { for } z \leq 12.5 \mathrm{~mm} \\
0 & \text { for } 12.5 \mathrm{~mm}<z \leq 37.5 \mathrm{~mm} . \\
\left(\frac{z / \mathrm{mm}-37.5}{5}\right)^{2} & \text { for } 37.5 \mathrm{~mm}<z\end{cases}
\end{aligned}
$$

$T_{\mathrm{SET}}$ is the set-point temperature for the crystal oven controller in degrees centigrade. With the mode propagation constants

$$
\begin{aligned}
\beta_{F}= & \frac{2 \pi}{1.32 \mu \mathrm{m}}\left[2.222701+9.8 \times 10^{-6}\left(\mathrm{~T} /{ }^{\circ} \mathrm{C}\right.\right. \\
& \left.-353.55)+6.66 \times 10^{-9}\left(\mathrm{~T} /{ }^{\circ} \mathrm{C}-353.55\right)^{2}\right], \\
\beta_{\mathrm{SH}}= & \frac{2 \pi}{0.66 \mu \mathrm{m}}\left[2.223597+100 \times 10^{-6}\left(T /{ }^{\circ} \mathrm{C}\right.\right. \\
& \left.-353.55)+75.55 \times 10^{-9}\left(\mathrm{~T} /{ }^{\circ} \mathrm{C}-353.55\right)^{2}\right]
\end{aligned}
$$

the wave-vector-mismatch distribution $2 \beta_{F}[T(z)]-$ $\beta_{\mathrm{SH}}[T(z)]$ was calculated.

The nonlinear switching was verified by two separate sets of measurements. First we kept the input intensity constant and ramped the temperature through phase matching so that the average wave-vector detuning and hence the magnitude of the effective nonlinearity was varied. The cascaded nonlinear phase
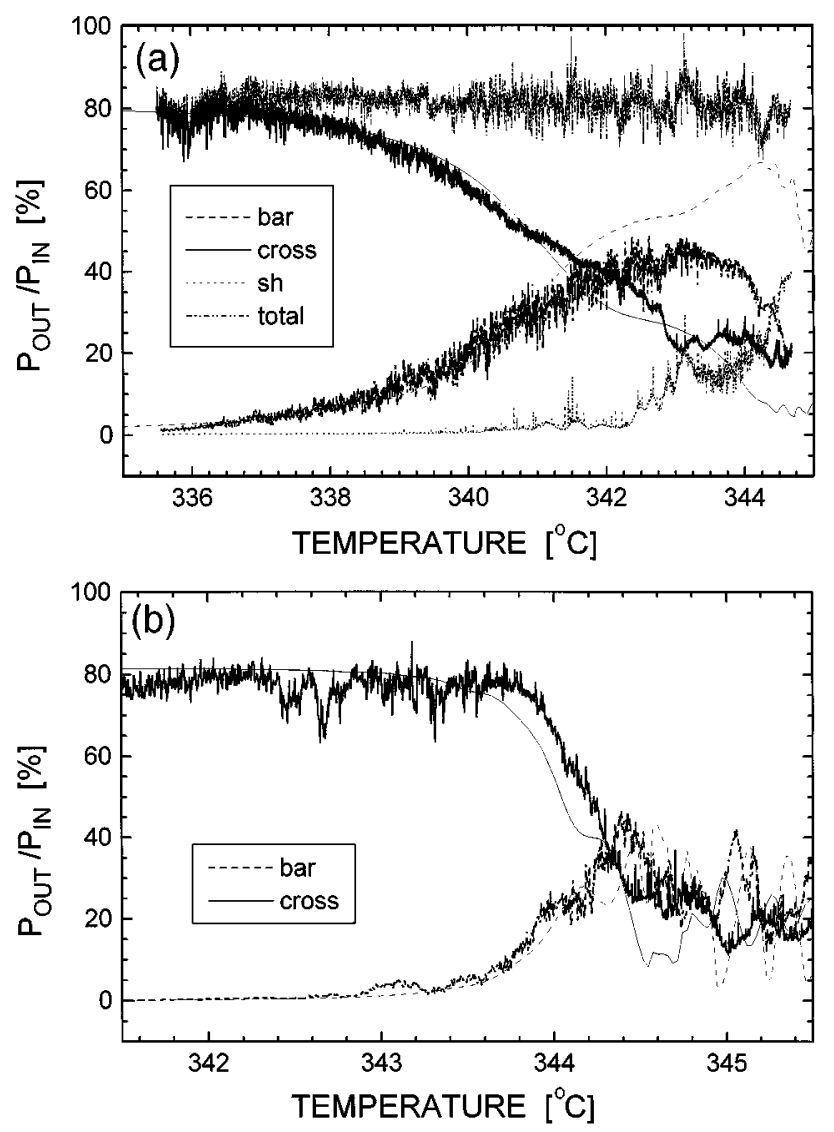

Fig. 1. Temperature dependence of the normalized output of a NLDC for inputs of (a) $6000 \mathrm{~W}$ and (b) $1500 \mathrm{~W}$. The smooth curves correspond to theory. sh, second harmonic. 

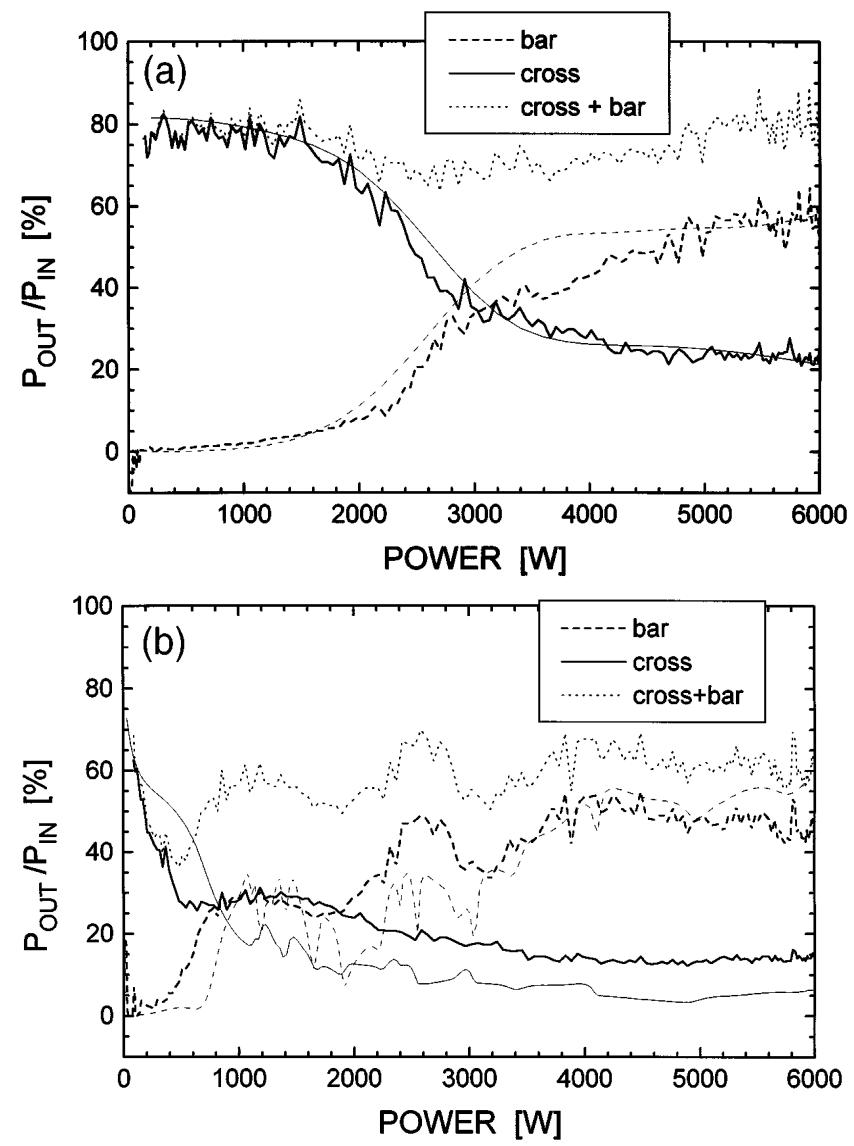

Fig. 2. Power dependence of a NLDC fundamental output for crystal temperatures of (a) $343.5^{\circ} \mathrm{C}$ and (b) $344.75^{\circ} \mathrm{C}$. The smooth curves correspond to theory.

shift induced a detuning between the coupler branches, reducing the power exchange between the waveguides. Figure 1 shows a typical result for a half-beat-length coupler. Far below the phase-matching temperature $T_{\mathrm{PM}} \approx 345^{\circ} \mathrm{C}$, weak cascading does not influence the coupling and, at the device output, all the incoupled light has transferred over into the cross branch. As the phase-matching temperature is approached from the low-temperature side (low-depletion region), the induced nonlinear phase shift decreases the power exchange; i.e., some light stays in the bar channel and the coupler begins to switch its output back to the bar channel with only a small loss owing to conversion into the second harmonic. For $T>T_{\mathrm{PM}}$ large harmonic generation and fundamental signal depletion occur. For lower input powers the same measurements showed that switching occurs at temperatures closer to $T_{\mathrm{PM}}$, where a larger cascaded nonlinearity provides the necessary nonlinear phase shift for switching, as expected.

In a second set of measurements we also measured the output dependence on the input power at a fixed temperature. Figure 2 shows typical results. In the temperature range where large phase shifts occur with small depletion, i.e., $T<T_{\mathrm{PM}}$, the switching curves are nearly identical to the switching curves obtained from $\chi^{(3)}$-based nonlinear directional couplers (NLDC's). ${ }^{4} \quad$ Figure 2(a) shows a switching curve at a crystal temperature of $343.5^{\circ} \mathrm{C}$, where fundamental depletion is still small. We observed an increase in the power required for switching when operating at temperatures even further below $T_{\mathrm{PM}}$, i.e., at larger average wave-vector mismatches and hence decreased cascading nonlinearity. Figure 2(b) shows a switching curve near phase matching, where depletion increases. As predicted in Ref. 9, we observe improved reduction in the cross channel with increasing input power, whereas the bar channel shows imperfect switch-up characteristics because of the generated second harmonic. The principal reason that the switching curves do not show a higher switching contrast at higher powers is that pulses were used, leading to some of the pulse breakup and to incomplete switching characteristics of $\chi^{(3)}$-based devices. All the experimental results are in good agreement with theory.

In summary, we have demonstrated that all-optical switching in a fully integrated NLDC is feasible with a cascaded second-order nonlinearity. It appears that the switching characteristics have some of the best switching ratios obtained to date in any material system. However, the current devices switch at peak powers orders of magnitude larger than those of the best $\chi^{(3)}$-based NLDC's. ${ }^{4}$ The goal here was to demonstrate that cascading could be used to implement highcontrast switching in a complicated device such as a NLDC with minimal loss to SHG. In the current implementation, the waveguides with their large effective core areas were not optimized to minimize the switching powers, and the geometry used in $\mathrm{LiNbO}_{3}$ has a relatively small value of $\chi^{(2)}$. For example, quasiphase-matched devices in $\mathrm{LiNbO}_{3}$ would reduce the peak switching powers by $\sim 2$ orders of magnitude and are planned for the future. Although even potentially larger nonlinearities are available, the problems of phase matching them have yet to be addressed.

The measurements were done at the Center for Research and Education in Optics and Lasers, University of Central Florida. The samples were prepared at the Universität Paderborn in Germany. The research was supported by the U.S. Army Reseach Office and the Advanced Research Projects Agency.

\section{References}

1. A. Armstrong, N. Bloembergen, J. Ducuing, and P. S. Pershan, Phys. Rev. 127, 1918 (1962).

2. R. Schiek, J. Opt. Soc. Am. B 10, 1848 (1993).

3. S. Singh, in Handbook of Laser Science and Technology, Supplement 2: Optical Materials, M. J. Weber, ed. (CRC, Boca Raton, Fla., 1995), Sec. 7.1, pp. 147-249.

4. A. Villeneuve, J. S. Aitchison, B. Vogele, R. Tapella, J. U. Kang, C. Trevinos, and G. I. Stegeman, Electron. Lett. 31, 549 (1995).

5. J. S. Aitchison, Y. Silberberg, A. M. Weiner, D. E. Leaird, M. K. Oliver, J. L. Jackel, E. M. Vogel, and P. W. E. Smith, J. Opt. Soc. Am. B 8, 1292 (1991).

6. R. Schiek, M. L. Sundheimer, D. Y. Kim, Y. Baek, G. I. Stegeman, H. Seibert, and W. Sohler, Opt. Lett. 19, 1949 (1994).

7. R. Schiek, Y. Baek, and G. I. Stegeman, Phys. Rev. E 53, 1138 (1996).

8. Y. Baek, R. Schiek, and G. I. Stegeman, Opt. Lett. 20, 2168 (1995)

9. R. Schiek, Opt. Quantum Electron. 26, 415 (1994). 